\title{
Avoiding Quantum Chaos in Quantum Computation
}

\author{
G.P. Berman $^{[a]}$, F. Borgonovi ${ }^{[b, c]}$, F.M. Izrailev ${ }^{[d]}$, V.I. Tsifrinovich ${ }^{[e]}$ \\ ${ }^{[a]}$ Theoretical Division and CNLS, Los Alamos National Laboratory, Los Alamos, New Mexico 87545 \\ ${ }^{[b]}$ Dipartimento di Matematica e Fisica, Università Cattolica, via Trieste 17, 25121 Brescia, Italy \\ [c] I.N.F.M., Gruppo Collegato di Brescia and I.N.F.N., Sezione di Pavia, Italy \\ ${ }^{[d]}$ Instituto de Fisica, Universidad Autonoma de Puebla, Apdo. Postal J-48, Puebla 72570, Mexico \\ [e] IDS Department, Polytechnic University, Six Metrotech Center, Brooklyn, NY 11201
}

\begin{abstract}
We study a one-dimensional chain of nuclear $1 / 2$-spins in an external time-dependent magnetic field. This model is considered as a possible candidate for experimental realization of quantum computation. According to general theory of interacting particles, one of the most dangerous effects is quantum chaos which can destroy the stability of quantum operations. The standard viewpoint is that the threshold for the onset of quantum chaos due to an interaction between spins (qubits) strongly decreases with an increase of the number of qubits. Contrary to this opinion, we show that the presence of a non-homogeneous magnetic field can strongly reduce quantum chaos effects. We give analytical estimates which explain this effect, together with numerical data supporting our analysis.
\end{abstract}

PACS numbers: 05.45Pq, 05.45Mt, 03.67,Lx

Much attention is paid in recent years to the idea of quantum computation (see, for example, [1] 3] and references therein). The burst of interest to this subject is caused by the discovery of fast quantum algorithm for the factorization of integers [4] demonstrating the effectiveness of quantum computers in comparison to the classical ones. Nowadays, there are different projects for the experimental realization of quantum computers, based on interacting two-level systems (qubits). One of the most important problems widely discussed in the literature, is the problem of decoherence which arises in many-qubit systems due to the influence of an environment [5]. However, even in the absence of the environment, the interaction between qubits may lead to the "internal decoherence" related to the onset of quantum chaos [6].

The latter subject of quantum chaos in closed systems of interacting particles has been developed recently in application to nuclear, atomic and solid state physics (see, e.g., [7] and references therein). When the (two-body) interaction between particles exceeds the critical value, fast transition to chaos occurs in the Hilbert space of manyparticle states [8]. Different aspects of this transition are now well understood, such as statistical description of eigenstates and the onset of thermalization in finite systems (see, e.g., [9] and references therein).

Direct application of the quantum chaos theory to a simple model of quantum computer [6] has shown that for a strong enough interaction between qubits the onset of quantum chaos is unavoidable. Although for $L=14-16$ qubits the critical value $J_{c r}$ for quantum chaos threshold is quite large, with an increase of $L$ it decreases as $J_{c r} \sim 1 / L$. From the viewpoint of the standard approach for closed systems of interacting particles, the decrease of the chaos threshold with an increase of qubits looks generic. However, in this Letter we demonstrate that this conclusion is not universal and the quantum chaos can be avoided, for example, with a proper choice of the external magnetic field.

Our consideration is based on the one-dimensional model of $L$ nuclear $1 / 2$-spins subjected to the timedependent magnetic field of the following form [10],

$$
\vec{B}(t)=\left[b_{\perp}^{p} \cos \left(\nu_{p} t+\varphi_{p}\right),-b_{\perp}^{p} \sin \left(\nu_{p} t+\varphi_{p}\right), B^{z}\right]
$$

where $B^{z}$ is a constant magnetic field oriented in the positive $z$-direction, $b_{\perp}^{p}, \nu_{p}$, and $\varphi_{p}$ are the amplitudes, frequencies and phases of a circular polarized magnetic field which is given by the sum of $p=1, \ldots, P$ rectangular pulses of the length $t_{p+1}-t_{p}$ rotating in the $(x, y)-$ plane, and providing a quantum computer protocol. The quantum Hamiltonian of this system has the form,

$$
\begin{aligned}
& \mathcal{H}=-\sum_{k=0}^{L-1}\left(\omega_{k} I_{k}^{z}+2 \sum_{n>k} J_{k, n} I_{k}^{z} I_{n}^{z}\right)- \\
& \frac{1}{2} \sum_{p=1}^{P} \Theta_{p}(t) \Omega_{p} \sum_{k=0}^{L-1}\left(e^{-i \nu_{p} t-i \varphi_{p}} I_{k}^{-}+e^{i \nu_{p} t+i \varphi_{p}} I_{k}^{+}\right),
\end{aligned}
$$

where the "pulse function" $\Theta_{p}(t)$ equals 1 only during the $p$-th pulse, for $t_{p}<t \leq t_{p+1}$. The quantities $J_{k, n}$ stand for the Ising interaction between two qubits, $\omega_{k}$ are the frequencies of spin's precession in the $B^{z}$-magnetic field, $\Omega_{p}$ is the Rabi frequency of the $p$ th pulse, $I_{k}^{x, y, z}=(1 / 2) \sigma_{k}^{x, y, z}$, the latter being the Pauli matrices, and $I_{k}^{ \pm}=I_{k}^{x} \pm i I_{k}^{y}$.

For the $p$-th pulse, the Hamiltonian (2) can be represented in the coordinate system which rotates with the frequency $\nu_{p}$. Thus, for the $p$-th pulse, our model can be reduced to the stationary Hamiltonian, 


$$
\begin{aligned}
& \mathcal{H}^{(p)}=-\sum_{k=0}^{L-1}\left[\left(\omega_{k}-\nu_{p}\right) I_{k}^{z}+\Omega_{p}\left(\cos \varphi_{p} I_{k}^{x}-\sin \varphi_{p} I_{k}^{y}\right)+\right. \\
& \left.2 \sum_{n>k} J_{k, n} I_{k}^{z} I_{n}^{z}\right],
\end{aligned}
$$

which describes the evolution for $t_{p}<t \leq t_{p+1}$.

The physically important regime of quantum computation is a selective excitation which corresponds to the range of parameters: $\Omega_{p} \ll J_{k, n} \ll \delta \omega_{k} \ll \omega_{k}$, where $\delta \omega_{k}=\left|\omega_{k+1}-\omega_{k}\right|[11]$. However, in this Letter, we consider a regime of non-selective excitation which is defined by the conditions, $\Omega_{p} \gg \delta \omega_{k} \gg J$, see details in [10]. This inequality provides the simplest way to prepare a homogeneous superposition of $2^{L}$ states needed for implementation of both Shor and Grover algorithms. In what follows we assume, for simplicity, that the amplitudes of magnetic pulses, and their frequencies and phases are equal, $\Omega_{p}=\Omega, \nu_{p}=\nu$, and $\varphi_{p}=\pi / 2$. Our main interest is in the nearest neighbor interaction $(N$ interaction) between qubits for two different cases, the dynamical one when all coupling elements are the same, $J_{k, n}=J \delta_{n, k+1}$, and the case when all values $J_{k, k+1}$ are random (random model). However, we will also discuss another case when all qubits interact to each other $(A$ interaction) with random $J_{k, n}$. In contrast to previously discussed model [6] with homogeneous magnetic field, below we consider the magnetic field with constant gradient along the z-axes. Therefore, we assume that the spin frequencies $\omega_{k}$ are slightly dependent on $k$ in the following way, $\delta \omega_{k} \ll \omega_{k}$. For the dynamical $N$-interaction and $\nu=\omega_{0}, \omega_{k}=\omega_{0}+a k(a>0)$, the Hamiltonian takes the form,

$$
H=\sum_{k=0}^{L-1}\left[-\delta_{k} I_{k}^{z}+\Omega I_{k}^{y}\right]-2 J \sum_{k=0}^{L-2} I_{k}^{z} I_{k+1}^{z} .
$$

where $\delta_{k}=a k$. In the $z$-representation the Hamiltonian matrix of size $N=2^{L}$ is diagonal for $\Omega=0$. For $\Omega \neq 0$, $H_{k, k+n}=i \Omega / 2$ and $H_{n k}=H_{k n}^{*}$. The matrix is very sparse, and it has quite specific structure if the basis is reordered according to an increase of the number $s$ written in the binary representation, $s=i_{1}, i_{2}, \ldots, i_{L}$ (with $i_{s}=0$ or 1 , depending on whether a single-particle state of $i$-th qubits is the ground state or the excited state).

The structure of eigenstates in this basis has been numerically studied in detail for $L=8,10,12$ in dependence on the strength of interaction, $J$, and different values of $\Omega$. For $\Omega \gg J$ the spectrum consists of $L+1$ narrow bands with large gaps of size $\approx \Omega$ between the bands. Since the most interesting energy region for preparation of the homogeneous wave function is in the middle of the energy (quasi-energy) spectrum, we consider below only the central band and the corresponding eigenstates for $L$ even.
In the absence of interaction between qubits, $J=0$, all eigenstates in the given basis are fully extended with the value of components $\psi_{n}$ close to $1 / \sqrt{N}$. Typical structure of eigenstates in the central band is shown in Fig. 1 for different values of $J$. One can see that with an increase of $J$-interaction, the probabilities, $w_{n}=\left|\psi_{n}\right|^{2}$, deviate from the unperturbed value, $w_{n}=1 / N$, thus resulting in quantum computation errors. The data demonstrate the transition from regular states for a weak interaction, $J \leq 0.1$, to chaotic ones for $J \approx 100$.
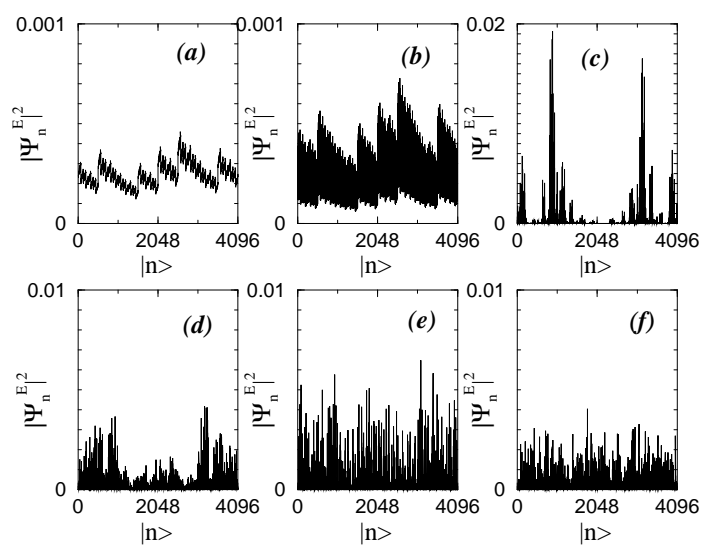

FIG. $1 . \quad$ Typical structure of eigenfunctions for $J=0,2 \cdot 10^{-4}, 0.1,1,10,100$ denoted by $(\mathrm{a}, \mathrm{b}, \mathrm{c}, \mathrm{d}, \mathrm{e}, \mathrm{f})$ respectively. Eigenstates are taken from the central energy band for $L=12, \Omega=100, a=1$.

Global properties of eigenstates can be characterized by the number, $N_{p c}$, of principal components, determined through the inverse participation ratio, $N_{p c}(E)=$ $\left[\sum_{n}\left|\psi_{n}(E)\right|^{4}\right]^{-1}$, where $\psi_{n}(E)$ is the $n$-th component of a specific eigenfunction. For zero interaction $(J=0)$, the number $N_{p c}$ is equal to $N$, and it decreases with an increase of interaction, thus, giving the measure of the destruction of unperturbed $(J=0)$ eigenstates. Note that for completely chaotic eigenstates with gaussian fluctua-

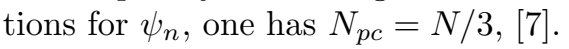

Numerical data for the averaged $N_{p c}$ in dependence on the interaction $J$ define different regions, see Fig. 2. The first region on the left of this figure corresponds to completely "delocalized" eigenstates with $\psi_{n} \approx 1 / \sqrt{N}$. Here the energy spectrum is characterized by many close quasidegenerate levels. In the second region where $N_{p c} \ll N$, all eigenstates are strongly influenced by the inter-qubit interaction. In this region which we denote as the region of weak chaos, the level spacing distribution $P(s)$ is quite close to the Poisson. From the data, the transition to the region of weak chaos occurs for $J=J_{c r} \approx 0.05$. One should stress that from the practical point of view the weak chaos should be avoided in the quantum computation because of large deviations of eigenstates from the unperturbed ones, see Fig. 1c-d.

Second transition to a strong quantum chaos occurs for 
$J \geq 20$. By the latter term we denote the situation when the level spacing distribution has the Wigner-Dyson form and fluctuations of components $\psi_{n}$ are close to the gaussian ones with $N_{p c} \approx N / 3$. Analysis shows that this transition corresponds to the overlapping of the central energy band to the nearest ones. As one can see, strong quantum chaos emerges for extremely strong interaction. Quite unexpectedly, the results for the random model (with random distribution of the interaction strength in the interval $[-J, J])$ turn out to be the same. This means that, both for the dynamical and random $N$-interactions our system is close to an integrable one for well separated energy bands.

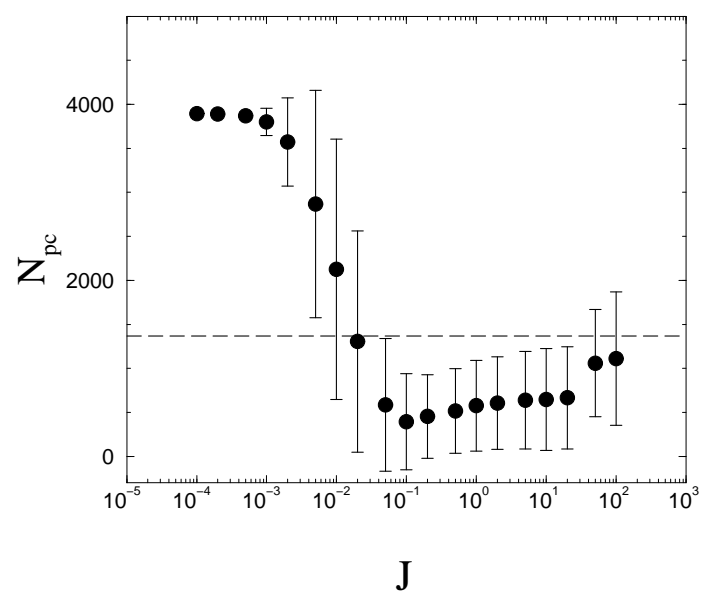

FIG. 2. The average number of principal components $N_{p c}$ as a function of $J$ for the parameters of Fig. If; the horizontal line corresponds to $N / 3$. The average is taken over the eigenfunctions from the central band only.

The above numerical analysis for $L=12$ shows that weak quantum chaos can significantly influence the structure of eigenstates, but the regime of strong chaos is not achievable in quantum computation. However, according to a common believe, the threshold for both weak and strong chaos is expected to decrease with an increase of $L$, thus, leading to the destruction of unperturbed states even in the presence of a very weak interaction $J$.

We show now analytically, that contrary to the standard point of view, in this model the chaos border $J_{c r}$ is independent on the number of qubits. In order to explain this unexpected phenomena, it is convenient to represent the Hamiltonian (4) in the basis in which it is diagonal for non-interacting $(J=0)$ qubits,

$$
H=H_{0}+J V_{0}
$$

Here the "effective field" Hamiltonian, $H_{0}$, is determined by the sum of $L$ individual Hamiltonians $H_{k}$,

$$
H_{0}=\sum_{k=0}^{L-1} H_{k}=\sum_{k=0}^{L-1} \sqrt{\left(\omega_{k}-\nu\right)^{2}+\Omega^{2}} I_{k}^{z}
$$

and the interaction $V_{0}=V_{\text {diag }}+V_{\text {band }}+V_{\text {off }}$ between new "quasi-particles" has the form,

$$
\begin{aligned}
& V_{\text {diag }}=-2 \sum_{k} b_{k} b_{k+1} I_{k}^{z} I_{k+1}^{z} ; \\
& V_{\text {band }}=-2 \sum_{k} a_{k} a_{k+1} I_{k}^{y} I_{k+1}^{y} ; \\
& V_{\text {off }}=2 \sum_{k}\left(a_{k} b_{k+1} I_{k}^{y} I_{k+1}^{z}+a_{k+1} b_{k} I_{k}^{z} I_{k+1}^{y}\right) .
\end{aligned}
$$

Three terms in the expression for $V_{0}$ stand for the diagonal, inside-band and between-bands interaction respectively, and

$$
b_{k}=\frac{\nu-\omega_{k}}{\sqrt{\left(\omega_{k}-\nu\right)^{2}+\Omega^{2}}} ; \quad a_{k}=\frac{\Omega}{\sqrt{\left(\omega_{k}-\nu\right)^{2}+\Omega^{2}}} .
$$

Under the condition, $\left|\omega_{k}-\nu\right| \ll \Omega$, we have the following expression for single-particle "quasi-energies" corresponding to the Hamiltonian $H_{0}$,

$$
\epsilon_{k}= \pm \frac{1}{2} \sqrt{\left(\omega_{k}-\nu\right)^{2}+\Omega^{2}}= \pm \frac{1}{2}\left(\Omega+\frac{a^{2} k^{2}}{2 \Omega}\right) .
$$

Note, that each "quasi-particle" can have $2 L$ different "quasi-energies" $\epsilon_{k}$ in contrast to actual particles (qubits) which have only two values of energy. The above expression allows us easily to construct many-particle unperturbed quasi-energies, $E_{c}=\sum_{k=0}^{L-1} \epsilon_{k}$, inside the central band. Indeed, since we have assumed $\Omega \gg\left|\omega_{k}-\nu\right|$ all many particles levels should have, for $L$ even, $L / 2$ positive $\epsilon_{k}$ and $L / 2$ negative ones. As a result, the total number $N_{c b}$ of many-body states in the central band is given by $N_{c b}=L !\left(\frac{L}{2} ! \frac{L}{2} !\right)^{-1}$ and the size can be estimate as twice the maximum energy, $(\Delta E)_{c b}=2 E_{c}^{(\max )}=$ $a^{2} L^{2}(L-1) / 8 \Omega$.

Now, we can estimate, for the $N$-interaction, the mean level spacing $\delta E$ between those many-body states which are directly coupled by the interaction, see Eq.(7). The value $\delta E$ can be estimated as the ratio $\delta E=$ $(\Delta E)_{f} / M_{f}$ where $M_{f} \approx L / 2$ is the number of manybody states coupled by $V_{\text {band }}$ (it is, in fact, the mean number per line of non-zero off-diagonal elements in the total Hamiltonian (5)). One should stress that the energy range $(\Delta E)_{f}$ within which these states are coupled, is less than the total energy width $(\Delta E)_{c b}$ of the central band. The value of $(\Delta E)_{f}$ can be estimated as the maximal difference between energies $E_{c}^{(2)}=\sum_{k}^{(2)} \epsilon_{k}$ and $E_{c}^{(1)}=\sum_{k}^{(1)} \epsilon_{k}$ of two many-body states $|1\rangle$ and $|2\rangle$ of $H_{0}$, that have the coupling $\left\langle 1\left|V_{\text {band }}\right| 2\right\rangle$ different from zero. From the expression (9) one finds, $(\Delta E)_{f}=2 a^{2} L / \Omega$. As a result, for $L \gg 1$ we have,

$$
\delta E=\frac{(\Delta E)_{f}}{M_{f}} \approx \frac{2 a^{2}}{\Omega}
$$

This mean spacing, $\delta E$, should be compared with the typical value of perturbation $V=J V_{0}$ [9]. The latter can 
be found from $V_{\text {band }}$ as $V \approx J / 2$. Therefore, we finally obtain,

$$
J_{c r} \approx \frac{4 a^{2}}{\Omega} .
$$

Remarkably, the threshold to chaos, $J_{c r}$, does not depend on the number $L$ of qubits. The origin of this phenomenon is that the width $(\Delta E)_{f}$ and $M_{f}$ both increase lineraly in $L$. This fact is entirely due to the quadratic growth of the energy shift in Eq. (9) for new singleparticles states, in dependence on $k$. Physically, this effect is related to the constant gradient of the magnetic field in the original Hamiltonian.

Numerical data for the number $N_{p c}$ of principal components of eigenstates inside the central energy band in the new basis (where $H_{0}$ is diagonal for $J=0$, see Eq. (5)) are given in Fig. 3 in dependence on $J / J_{c r}$. It is clearly seen that below the border, $J<J_{c r}$, there is a scaling dependence of $N_{p c}$ on $L$ and $\Omega$ which confirms our estimate (11). On the other side, for $J>J_{c r}$, the value of $N_{p c}$ saturates to its maximal value $N_{c b} / 3$ in correspondence with random matrix predictions [7]. We notice that the transition to extended states in the Hamiltonian (5), which occurs for $J>J_{c r}$, corresponds to the transition to the weak chaos given in Fig.2.

In the case of random model, the data turns out to be similar, thus, confirming our conclusion that for the $N$-interaction the model is close to an integrable one, independently on whether the interaction is dynamical or random. One should note that, indeed, if one neglects the interband interaction $V_{o f f}$, the Hamiltonian (5) is integrable, as shown in 13. Thus, our analysis shows that the border $J_{c r}$ does not decrease with an increase of number of qubits.

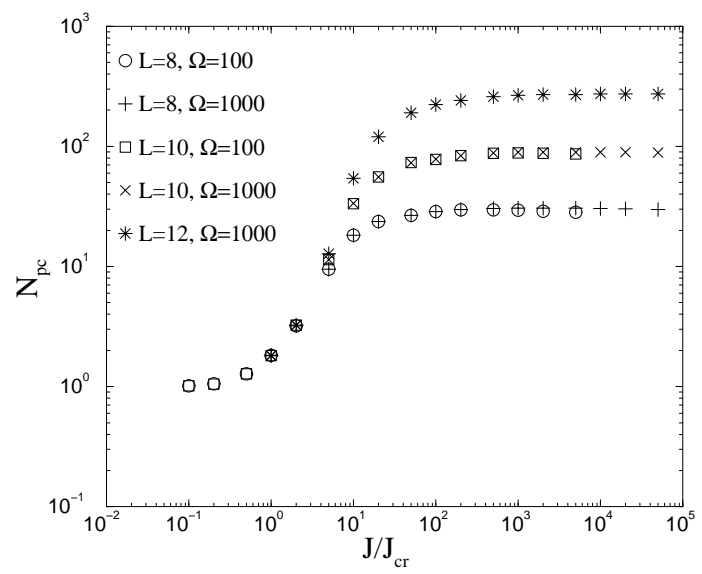

FIG. 3. The average number of principal components for the dynamical $N$-interaction as a function of $J / J_{c r}$, in the central band in the $H_{0}$-basis, for $L=8,10,12$ and different values of $\Omega$.

Now we have to take into account that with an increase of $L$, the width $(\Delta E)_{c b}$ of the central band can exceed the distance $\Omega$ between the nearest ones, which leads to strong quantum chaos. The estimate for the critical value, $L_{c r}$, which corresponds to the band overlapping in absense of the $J$-interaction reads as $L_{\max } \sim$ $(\Omega / a)^{2 / 3} \gg 1$.

For the model with random interaction between all qubits ( $A$-interaction), strong quantum chaos occurs inside the energy bands manifested by a Wigner-Dyson distribution for $P(s)$ (more details will be given elsewhere). In this case the estimate for the energy width $(\Delta E)_{f}$, within which many-body states are coupled by the twobody interaction, is $(\Delta E)_{f}=L^{2} / 2 \Omega$, and $M_{f}=a^{2} L^{2} / 4$. Therefore, we get $\delta E=(\Delta E)_{f} / M_{f} \approx 2 a^{2} / \Omega$ which is the same as for the $N$-interaction. This is an unexpected result since generically, the chaos border for $A$-interaction is much less than for $N$-interaction $\left(J_{c r} \propto 1 / L^{2}\right.$, see, e.g. [12]). Numerical data for the $A$-interaction in (4) confirm the above result (the corresponding figure is very similar to Fig. 3).

In conclusion, we have shown that, in contrast to general believe, the chaos border in the model of $L$ interacting qubits does not decrease with an increase of $L$, in the presence of a strong magnetic field with constant gradient in the $z$-direction. The quantum chaos which emerges for a very strong interaction between qubits is irrelevant to quantum computation. The mechanism of strong chaos is shown due to the band overlapping only, and can be avoided even for a very large number of qubits. Moreover, our analysis shows that for an inhomogeneous gradient of magnetic field (for example, when $\omega_{k} \propto k^{4}$ ), one can expect that the border for chaos increases with an increase of $L$. The region of parameters for quantum computation with selective excitation requires additional analysis.

The work of GPB and VIT was supported by the Department of Energy (DOE) under the contract W-7405ENG-36, by the National Security Agency (NSA) and Advanced Research and Development Activity (ARDA). FMI acknowledges the support by CONACyT (Mexico) Grant No. 34668-E.

[1] A. Steane, Rep. Progr. Phys., 61, 117 (1998).

[2] G.P. Berman, G.D. Doolen, R. Mainieri, and V.I. Tsifrinovich, Introduction to Quantum Computers, World Scientific Publishing Company, 1998.

[3] M.A. Nielsen, I.L. Chuang, Quantum Computation and Quantum Information, Camgridge University Press, 2000.

[4] P. Shor, Proc. of the 35th Annual Symposium on the Fundations of Computer Science, IEEE, Computer Society Press, New York, (1994), p.124.

[5] I.L. Chuang, R. Laflamme, P.W. Shor, and W.H. Zurek, Science, 270, 1633 (1995). 
[6] B. Georgeot and D.L. Shepelyansky, Phys. Rev. E., 62, 3504 (2000); ibid, 6366.

[7] T. Guhr, A. Müller-Groeling, and H.A. Weidenmïler, Phys. Rep., 200, 189 (1999).

[8] B.L. Altshuler, Y. Gefen, A. Kamenev and L.S. Levitov, Phys. Rev. Lett., 78, 2803 (1997).

[9] V.V. Flambaum and F.M. Izrailev, Phys. Rev. E 56, 5144 (1997); F.M. Izrailev, in Proceedings of the International School of Physics "Enrico Fermi", Course CXLIII, Varenna 20-30 July, eds. G.Casati, I.Guarneri and U.Smilansky, IOS Press, 2000, pp.371-430.

[10] G.P. Berman, F. Borgonovi, F.M. Izrailev, and V.I. Tsifrinovich, quant-ph/0006095.

[11] G.P. Berman, G.D. Doolen, G.V. Lopez, V.I. Tsifrinovich, Phys. Rev. B, 61, 2305 (2000); Phys. Rev. A, 61, 2307 (2000).

[12] B. Georgeot and D. Shepelyansky, Phys. Rev. Lett., 81, 5129 (1998).

[13] A.P. Young, Phys. Rev. B, 56, 11691 (1997). 